\title{
Expanding the Horizon of Molecular Gas Surveys of Nearby Galaxies
}

\author{
Zhong Wang, Xuejian Jiang, and Linda Watson \\ Smithsonian Astrophysical Observatory email: zwang@cfa.harvard.edu
}

\begin{abstract}
This contribution discusses the prospect of using millimeter interferometry to survey molecular gas in a large number of galaxies beyond the distance of Virgo.
\end{abstract}

Keywords. galaxies: ISM - techniques: interferometric

\section{Observing Molecular Gas in A Large Galaxy Sample}

Galaxies forming stars continuously need a steady supply of gas to sustain its activities, so tracing the ISM contents of galaxies is an effective way to follow galaxy evolution and examine its relationship to the environment. The ALFALFA survey program has measured the HI 21-cm line of tens of thousands of gas-rich galaxies, with distances up to $250 \mathrm{Mpc}$ (Haynes et al. 2011). A rich complementary dataset from UV, visible to infrared are also being assembled for this galaxy sample.

For galaxies in the distance range beyond Virgo, single dish measurements of molecular (CO) emissions in the millimeter wavelength may be ineffective due to beam dilution. In contrast, aperture synthesis observations can be configured to optimize the use of the antenna's collecting areas and maximize the signal-to-noise ratio. Furthermore, with a large parent sample such as the ALFALFA, track-sharing of targets and automated backend tuning can be used so that a sizeable survey can be carried out efficiently.

\section{Results of Test Observations}

We have conducted test observing runs with the SMA over the last few months based on the ALFALFA sample. Our tests suggest at 5-sigma level, we can reach a $\mathrm{CO}(2-1)$ line flux limit of $6 \mathrm{Jy} \mathrm{km} \mathrm{s}^{-1}$ in a 2-hour integration (using $20 \mathrm{~km} \mathrm{~s}^{-1}$ bins and assuming an average line width) with the SMA in its subcompact configuration under median observing conditions ( $\mathrm{pwv} \leqslant 4 \mathrm{~mm}$ ). This corresponds to molecular gas masses ranging from $10^{7}$ to $10^{9} \mathrm{M}_{\odot}$ depending on the redshift of the galaxy, but generally at the $\leqslant 10 \%$ level of HI gas masses of the ALFALFA sample.

Some follow-ups CO observations to the ALFALFA have been conducted elsewhere, but single-dish observations have been limited to the most massive subsample of ALFALFA galaxies (e.g., Saintonge et al. 2011a, b), while observing at higher frequencies $(345 \mathrm{GHz})$ are relatively costly in terms of telescope time. If access to a substantial amount of 230 $\mathrm{GHz}$ interferometric observing time can be obtained, it will pave the way for an survey program that considerably exceeds the current limitations.

\section{References}

Haynes, M. P., et al. 2011, AJ, 142, 170

Saintonge, A., et al. 2011a, b, MNRAS, 415, 32 and 61 